\title{
Altered Regional Brain Functional Activity Predominantly Involving the Right Superior Temporal Gyrus in Patients With Vestibular Migraine Diagnosed According to the Diagnostic Criteria of the Bárány Society and the International Headache Society
}

Zhe-Yuan Li

Aerospace center hospital

Li-Hong Si

Aerospace center hospital

Bo Shen

Aerospace center hospital

Xia Ling

Aerospace center hospital

Xu Yang ( $\nabla$ angxu2011@163.com )

Aerospace central hospital

Research article

Keywords: vestibular migraine, spontaneous brain activity, functional connectivity, ALFF, fALFF, ReHo

Posted Date: April 30th, 2021

DOI: https://doi.org/10.21203/rs.3.rs-467377/v1

License: (c) (i) This work is licensed under a Creative Commons Attribution 4.0 International License. Read Full License 


\section{Abstract}

Background: Vestibular migraine (VM) is considered one of the most common cause of episodic central vestibular disorders, the mechanism of VM is currently still unclear. It is worth investigating whether VM belongs to the migraine subtype or is a separate disorder. The development of functional nuclear magnetic resonance (fMRI) in recent years offers the possibility to explore the pathogenesis of VM in depth. The study aimed to investigate resting-state functional brain activity alterations in patients with VM diagnosed based on the diagnostic criteria of the Bárány Society and the International Headache Society.

Methods: Seventeen patients with VM who received treatment in our hospital from December 2018 to December 2020 were enrolled. Clinical data of all patients were collected. Eight patients with migraine and 17 health controls (HCs) were also included. All subjects underwent $\mathrm{fMRI}$ examination. The amplitude of low frequency fluctuation (ALFF), fractional amplitude of low frequency fluctuation (fALFF) and regional homogeneity (ReHo) were calculated to observe the changes in spontaneous brain activity in patients with VM. Then brain regions with altered spontaneous brain activity were selected for seeded-based functional connectivity (FC) analysis to explore the changes in $\mathrm{FC}$ in patients with VM.

Results: Among 17 patients with VM, there were 7 males and 10 females with an average age of $39.47 \pm 9.78$ years old. All patients with VM had a history of migraine. Twelve (70.6\%) patients with VM had recurrent spontaneous vertigo, 2 (11.7\%) patients had visually-induced vertigo, and 3 (17.6\%) patients had head motion-induced vertigo. All 17 patients with VM reported worsening of dizziness vertigo during visual stimulation. The migraine-like symptoms were photophobia or phonophobia $(n=15,88.2 \%)$, migraine-like headache $(n=8,47.1 \%)$, visual aura during VM onset $(n=7,41.2 \%) .5(29.4 \%)$ patients with VM had hyperactive response during the caloric test, and $12(70.6 \%)$ patients had caloric test intolerance. Eleven (64.7\%) patients had a history of motion sickness. VM patients showed exhibited significantly increased ALFF and fALFF values in the right temporal lobe (STG and MTG), and significantly increased ReHo values in the right STG, MTG and ITG in comparison with HCs. Compared with patients with migraine, patients with VM showed significantly decreased ALFF values in the right median cingulate and paracingulate gyri, significantly increased fALFF values in the right parietal lobe (postcentral gyrus and superior parietal gyrus), and the right frontal lobe (supplementary motor areas and dorsolateral superior frontal gyrus), as well as significantly increased ReHo values in the right thalamus. Compared with HCs, patients with migraine showed significantly increased ALFF values in the right limbic lobe (right parahippocampal gyrus and right fusiform gyrus), left ITG and the right frontal lobe (supplementary motor areas, right median cingulate and paracingulate gyri, and right right inferior frontal gyrus), significantly decreased ALFF values in the pons and brainstem, significantly decreased ReHo values in the frontal cortex (including left and right supplementary motor areas, left dorsolateral superior frontal gyrus, left median cingulate and paracingulate gyri, right paracentral lobule, right dorsolateral superior frontal gyrus, left and right middle frontal gyrus).

Conclusions: Ventral stream of visual processing and allocentric spatial cognition in patients with VM may be impaired. Vertigo attacks in patients with VM may be related to increased spontaneous activity in the right parietal lobe-frontal lobe-thalamus; patients with VM and migraine both had altered brain function, but the underlying mechanism seems to be different.

\section{Introduction}


Vestibular migraine (VM) is considered the most common cause of central episodic vertigo, accounting for $7 \%$ of patients seen in neuro-otological clinics and 9\% of patients seen in headache clinics [1]. In 2012, diagnostic criteria for VM was jointly formulated by the Bárány Society and the Migraine Classification Subcommittee of the International Headache Society, which were published in the International Classification of Headache Disorders, 3rd edition (beta version) (ICHD-III beta) in 2013 [2]. At present, the clinical diagnosis of VM still only relies on clinical symptoms [3]. During VM attacks, patients usually have abnormalities in perception of voluntary movement, such as rotation or swing, which are often accompanied by increased visual motion sensitivity and spatial disorientation [4]. Sometimes even during the interictal period, nystagmus may be observed in patients, suggesting that there is a certain degree of brain stem involvement [5]. Among the hypotheses about the mechanisms of VM, most hypotheses categorize VM as a subcategory of migraine and suggest that there is overlap among VM and vestibular pathway damage. However, the current vestibular testing techniques are not yet able to make the differentiation of VM from other vestibular diseases [6], there is still debate as to whether VM is central or peripheral in origin $[7,8]$.

With the development of functional imaging techniques, such as the application of positron emission tomography (PET) and functional nuclear magnetic resonance ( $\mathrm{fMRI}$ ) provides a possibility of studying the mechanism of VM. Previous studies have shown that patients with VM often have abnormal vestibular information processing and abnormal functional interactions between visual and vestibular networks in the cerebral cortex during tilt $[9,10]$. Shin et al. [10] performed 8F-deoxyglucose PET study of two patients with VM during the interictal and ictal period, and found that compared with interictal period of VM, patients showed increased metabolism in the bilateral cerebellum, frontal cortices, temporal cortex, posterior insula, and thalami, and decreased metabolism in the occipital cortex during the ictal period of VM, increased metabolism in the temporo-parieto-insular areas and bilateral thalami indicates the activation of the vestibulo-thalamo-cortical pathway, decreased metabolism in the occipital cortex suggests reciprocal inhibition between the visual and vestibular systems. These findings revealed that metabolic activities in the visual and vestibular cortical areas were altered during acute VM attack.

In previous studies [11,12], scholars have used task fMRI data from different tasks to investigate VM. A task-state fMRI study [11] in healthy volunteers exposed to magnetic vestibular stimulation showed that the brain regions involved in balance and spatial navigation are complex networks, including the insula, superior temporal gyrus (STG) and inferior parietal lobule, precentral gyrus and postcentral gyrus, anterior insula and inferior frontal gyrus, anterior cingulate gyrus and hippocampus. Russo et al. [12] performed a fMRI study in 12 patients with VM during ear irrigation with cold water and found that patients with VM showed significantly increased activation in the left dorsal thalamus during left-sided vestibular stimulation, and the authors speculated that the pain and vestibular sense in patients with VM may be related to the thalamus $[13,14]$.

At present, fMRI studies on VM patients are mostly task-based, and most study showed that activation of multilevel vestibular integrating centers and cortical areas related to higher-level vestibular information processing is present in patients with VM. However, task-related brain regions are sometimes not associated with brain functional changes related to VM itself, which may be associated with brain functional changes related to task stimulation. Therefore, the real-time measurement of spontaneous brain functional activities by using restingstate fMRI can better reflect the pathogenesis of VM [15].

Under this background, we investigated the changes in resting-state brain spontaneous activity and functional connectivity (FC) between patients with $\mathrm{VM}$, health controls ( $\mathrm{HCs}$ ) and patients with migraine by using restingstate regional brain activity measures including amplitude of low frequency fluctuation (ALFF), fractional 
amplitude of low frequency fluctuation (fALFF), regional homogeneity (ReHo), and seed-based functional connectivity analysis in this study, and aimed to explore functional brain activity alterations in patients with VM and its possible mechanisms involving the vestibular pathway.

\section{Methods}

\section{Study subjects}

A total of 17 patients with VM who received treatment in Aerospace Center Hospital, Peking University Aerospace School of Clinical Medicine between December 2018 and December 2020 were included. Clinical data of all patients were collected. Blood pressure, cardiac function, thyroid function tests, immune-related laboratory tests and lower limb electromyography were performed to exclude other medical diseases. Videonystagmography, caloric test, head impulse test, and vestibular evoked myogenic potential stimulation were performed to exclude peripheral vestibular lesions. MRI was performed to exclude severe neurological diseases.VM was diagnosed based on Bárány Society's diagnostic criteria (3) as following: (A) at least 5 episodes of vestibular symptoms of moderate or severe intensity lasting 5 minutes to 72 hours; $(B)$ current or previous history of migraine with or without aura according to the diagnostic criteria of ICHD; (C) one or more of the following migraine features with at least $50 \%$ of the vestibular episodes: $50 \%$ of vestibular attacks are accompanied by at least one migraine symptom: (1) headache with at least two of the following characteristics: one sided location, pulsating quality, moderate or severe pain intensity, aggravation by routine activity; (2) photophobia and phonophobia; (3) visual aura; (D) not better accounted for any other vestibular or IHCD diagnosis.

17 age- and gender-matched HCs were included, they had no history of headache or vertigo and had no severe medical diseases. 8 age- and gender-matched patients with migraine were also included in the study, patients with migraine was diagnosed according to the diagnostic criteria of ICHD-III $\beta$ in 2013 [16], patients with migraine had no history of vertigo and had no severe medical diseases.

All subjects underwent peripheral vestibular function evaluation to exclude peripheral vestibular lesions. Patients with VM (during an interictal phase), HCs, and patients with migraine (during an interictal phase) were further scanned for fMRI. This study was approved by the Ethics Committee of Aerospace Center Hospital, Peking University Aerospace School of Clinical Medicine. All subjects volunteered to participate in this study and signed informed consent.

During the caloric test, the left and right external auditory canals were irrigated with cold $\left(30^{\circ} \mathrm{C}\right)$ and warm $\left(44^{\circ} \mathrm{C}\right)$ water with the patient in the supine position and their head raised by $30^{\circ}$. The right external auditory canal was first irrigated with warm water, followed by the left external auditory canal with warm water and the right external auditory canal with cold water, and last the left external auditory canal with cold water (i.e., right warm (RW), followed by left warm (LW) and right cold (RC), and last left cold (LC)). There was a 5-min interval between irrigations. The slow-phase velocity (SPV) during irrigation was recorded, and the canal paresis (CP) value was calculated. A CP value of $>25 \%$ indicates reduced unilateral horizontal semicircular canal function [17]. A sum of the SPV values of the bilateral semicircular canals of $\leq 12 \%$ s suggests reduced bilateral horizontal semicircular canal function. The criteria for hyperactive responses were as follows: total peak cool response (LC + RC) of $>99^{\circ} / \mathrm{s}$, total peak warm response $(\mathrm{LW}+\mathrm{RW})$ of $>146^{\circ} / \mathrm{s}$, total peak response $(\mathrm{LC}+\mathrm{RC}+\mathrm{LW}+\mathrm{RW})$ of $>221^{\circ} / \mathrm{s}$. Caloric test intolerance refers to the main symptoms, including obvious nausea, vomiting, numbness in hands and feet, and body stiffness. Caloric test intolerance was considered severe if its duration was $>1 \mathrm{~h}$. 


\section{Image acquisition}

All subjects were scanned using a 3.0-Tesla MR (SIEMENS MAGNETOM Skyra syngo MR D13, Germany) with a 16-channel head and neck coil. During image acquisition, subjects' head was immobilized to avoid head movement. Subjects were asked to relax with their eyes closed, stay awake throughout the scanning. Images was obtained in sagittal plane using a 3D gradient-echo T1WI sequence with the following parameters: 192 sagittal slices; repetition time $=1900 \mathrm{~ms}$; echo time $=2.43 \mathrm{~ms}$; flip angle $=8^{\circ}$; field of view $=256 \mathrm{~mm} \times 256 \mathrm{~mm} \times 256 \mathrm{~mm}$; Voxel size $=1.0 \mathrm{~mm} \times 1.0 \mathrm{~mm} \times 1.0 \mathrm{~mm}$. Resting-state $\mathrm{fMRI}$ images was obtained using echo-planar imaging (EPI) sequence sequence: repetition time $=2000 \mathrm{~ms}$; echo time $=30 \mathrm{~ms}$; flip angle $=90^{\circ}$; field of view $=222 \mathrm{~mm} \times 222$ $\mathrm{mm} \times 222 \mathrm{~mm}$; Voxel size: $3.0 \mathrm{~mm} \times 3.0 \mathrm{~mm} \times 3.0 \mathrm{~mm}$. After scanning, a total of 200 volumes were obtained, and the scan time was 6 minutes and 48 seconds. All subjects remained awake during scanning and did not experience significant discomfort during or after scanning.

\section{MRI image data processing}

Image data were processed using DPARSFA software on the MATLAB2013 platform,

\section{ALFF analyisis}

ALFF analysis was done with following steps: (1) data conversion: image data were converted from DICOM format to NIFTI format that can be processed by SPM12; (2) removal of the first 10 time points: due to the possible instability of the initial MRI signal caused by T1 relaxation and the need for participants to adapt to the scanning environment, the first 10 time points were deleted; (3) time correction: time correction is performed to ensure that all voxels within one volume had been acquired at the same time, the 35th slice was chosen as the reference slice; (4) head movement correction: slight head motion of the subject between the time points during the scan was corrected, in order to ensure the accuracy of the position information, the subjects who had more than $1.5 \mathrm{~mm}$ head translation in $\mathrm{x}-\mathrm{y}$-, or $\mathrm{z}$-direction and $1.5^{\circ}$ head rotation were removed; (5) removal of linear drift: linear trend formed due to heating of MR scanner and fatigue in subjects after long MRI scanning time was removed; (6) regression: a linear regression model was used to remove the interference signal in the BOLD signal; (7) spatial normalization: to solve the problems related to difference in brain morphology among different subjects and the inconsistencies in spatial position during scanning, $\mathrm{fMRI}$ images were spatially normalized to a standard space using DARTEL and resampled at a resolution of $3 \mathrm{~mm} \times 3 \mathrm{~mm} \times 3 \mathrm{~mm}$; (8) spatial smoothing: smoothening was conducted with a Gaussian kernel of $8 \mathrm{~mm} \times 8 \mathrm{~mm} \times 8 \mathrm{~mm}$ to reduce registration errors and increase the normality of the data; (9) ALFF calculation: the power spectrum is obtained by converting signals in time domain into the frequency domain using the Fast Fourier Transform, the average square root of the power spectrum was calculated as the ALFF value; (9) fALFF calculation: fALFF value was calculated at frequency range of $0.01-0.1 \mathrm{~Hz}$.

\section{REHO}

ReHo analysis was performed with following steps: (1) data format conversion; (2) removal of first 10 time points; (3) time correction; (4) head movement correction; (5) linear drift removal; (6) regression; (7) band-pass filtering: the time series for each voxel was filtered (band-pass, 0.01-0.8 Hz) to remove low-frequency drift and high frequency noise; (8) spatial normalization; (9) REHO calculation; (10) spatial smoothing.

\section{Seed-based FC analysis}


Seed-based FC analysis was performed with following steps: (1) data format conversion; (2) removal of first 10 frames; (3) time correction; (4) head movement correction; (5) linear drift removal; (6) regression; (7) band-pass filtering; (8) spatial normalization; (9) spatial smoothing; (10) FC calculation: brain regions with altered fALFF were converted into MASKs using Xjview, and selected as seeds for seeded-based FC analysis to assess FC

alterations with other brain regions, particularly especially other vestibular cortex and visual cortex areas.

\section{Statistical analysis}

fMRI data analysis was performed using SPM 12 software. Comparison of the difference in results of ALFF, REHO, FC between groups were tested using a two-sample $T$ test and adjusted for covariates including age and sex. $P<$ 0.01 was considered to be statistically different. Multiple comparisons corrections were performed using familywise-error correction (FWE). Results were visualized using XJVIEW and BrainNet.

\section{Results}

\section{Clinical data of patients included in the study}

In the VM group, there are 7 males and 10 females, all were right-handed, and the average age was $39.47 \pm 9.78$ years. The median length of medical history was 5 years (range,1-30 years). As to the frequency of VM attacks, one patient reported one attack per week, 7 patients reported one attack per month, 9 patients reported once attack per year. In the HC group, there were 7 males and 10 females with an average age of $39.82 \pm 13.01$ years, all $\mathrm{HCs}$ were right-handed. In the MC group, there were 3 males and 5 females, all were right-handed, the average age was $41.88 \pm 11.03$ years.

All 17 (100\%) patients with VM had a history of migraine, including 12 (70.6\%) patients of recurrent spontaneous vertigo, 2 (11.7\%) patients of visually-induced vertigo, and 3 (17.6\%) patients of head motion-induced vertigo. All 17 patients with VM reported worsening of dizziness during visual stimulation. The accompanying symptoms were photophobia or phonophobia $(n=15,88.2 \%)$, migraine-like headache $(n=8,47.1 \%)$, visual aura during VM attacks $(n=7,41.2 \%)$.

During the caloric test, hyperactive response was found in 5 (29.4\%) patients with VM, and caloric test intolerance was found in 12 (70.6\%) patients. Among 17 patients with VM, 11 (64.7\%) patients had a history of motion sickness (Table 1).

\section{ALFF and fALFF results}

Compared with HCs, VM group exhibited significantly increased ALFF and fALFF values in the right temporal lobe, including STG and middle temporal gyrus (MTG) ( $X=51, Y=-54, Z=6, P=0.002$, FWE corrected; $X=69, Y=-45, Z=3$; $\mathrm{P}=0.001$, FWE corrected, respectively, Table 2). ALFF and fALFF refer to the intensity of spontaneous brain activity. The higher the values, the greater the spontaneous brain activity in the brain regions. Increased ALFF values in the right temporal lobe in patients with VM indicated that the intensity of spontaneous functional activity in this region was enhanced.

Compared with migraine group, VM group showed significantly decreased ALFF values in the right median cingulate and paracingulate gyri $(X=21, Y=-30, Z=45, P=0.009$, FWE corrected, Table 2), indicating that the intensity of spontaneous functional activity in this region in patients with VM was weakened. VM group also 
showed significantly increased fALFF values in the right parietal lobe (postcentral gyrus and superior parietal gyrus) ( $X=24, Y=-45, Z=69, P=0.011$, FWE corrected, Table 2), and right frontal lobe (supplementary motor area (SMA) and dorsolateral superior frontal gyrus) $(X=24, Y=-45, Z=69, P=0.026$, FWE corrected, Table 2) compared with migraine group. The results indicated that the intensity of spontaneous functional activity in the abovementioned brain regions in patients with VM was increased.

Compared with the HC group, migraine group showed significantly increased ALFF values in the right limbic lobe (mainly including the right parahippocampal gyrus and right fusiform gyrus) ( $X=33, Y=-18, Z=-33, P=0.04, F W E$ corrected), left temporal lobe (left inferior temporal gyrus (ITG)) (X=57, Y=-36, Z =-27, $P=0.024$, FWE corrected) and the right frontal lobe (mainly including SMA, right median cingulate and paracingulate gyri, and right inferior frontal gyrus) $(X=21, Y=-18, Z=54)$, as well as significantly decreased ALFF values in the pons and brainstem (Table 2).

\section{ReHo results}

Patients with VM showed increased ReHo values in the right temporal lobe (STG, MTG and ITG) in comparison with HCs (X=60, Y=-45, Z=6, P=0.013, FWE corrected, Table 2). ReHo refers to the synchronization of spontaneous functional activities among an individual voxel and surrounding voxels. Increased ReHo values in the right temporal lobe indicated an increase in consistency of regional brain activity in this region in patients with VM. Regions with increased ReHo values contain regions with increased ALFF values and adjacent regions. Increased ReHo values and ALFF values were both observed in the right temporal lobe, indicating that the regional functional activities in the right temporal lobe (STG, MTG, and ITG) in patients with VM were enhanced.

Patients with VM showed significantly increased ReHo values in the right thalamus compared with patients with migraine $(X=12, Y=-21, Z=-3, P=0.043$, FWE corrected, Table 2)), suggesting an increase in consistency of regional brain activity in this region in patients with VM.

Compared with HCs, patients with migraine had significantly decreased ReHo values in the frontal cortex (mainly including left and right SMA, left dorsolateral superior frontal gyrus, left median cingulate and paracingulate gyri, right paracentral lobule, right dorsolateral superior frontal gyrus, left and right middle frontal gyrus) $(X=-18, Y=3$, $\mathrm{Z}=60, \mathrm{P}<0.001$, FWE corrected, Table 2).

\section{Results from seed-based FC analysis}

Brain regions with altered fALFF were selected for seeded-based FC analysis. The results revealed significantly increased FC in the right temporal lobe (STG and MTG), small part of the parietal lobe (inferior parietal lobe) and the insular lobe in patients with VM compared with the HC group ( $X=51, Y=-45, Z=15, P=0.016$, FWE corrected). No significant difference was found between migraine and $\mathrm{HC}$ groups, as well as VM and migraine groups.

\section{Discussion}

VM is one of the common causes of episodic central vestibular disorders, which affects more than $1 \%$ of the general population [18]. Generally, diagnosis of VM is only based on history of vertigo and migraine, and there is still a lack of more accurate laboratory tests and objective evidence that supports the diagnosis. The underlying mechanisms of VM are still unclear. Whether vestibular migraine belongs to the migraine subtype or is a separate disorder deserves further study. 
Clinically, the vestibular symptoms are very prominent in patients with VM compared to patients with migraine. In this study, we found that majority of patients with VM (12/17) had spontaneous vertigo, which was mostly triggered by head or visual movements. The result is similar to the findings of Neuhauser et al [19] and Cho et al [20]. In fact, the heterogeneity of vestibular symptoms may be related to patients' subjective description. In this study, it is interesting that most patients with VM (70.6\%) developed caloric test intolerance, this may be due to increased sensitivity of the vestibule $[21,22]$. Caloric test intolerance has been repeatedly reported in previous studies, patients usually developed severe intolerance during irrigation with warm or cold water, such as obvious nausea, vomiting, heart palpitations, numbness of hands and feet, and even body stiffness. In some patients, those symptoms may even take several hours to recover [5, 6, 23-25]. In addition, we found that $64.7 \%$ patients with VM had motion sickness. The correlation between motion sickness and VM has been reported by Akdal et al. [26], their findings showed that motion sickness was more common in migraine accompanied by vertigo compared with patients with migraine (75\% vs 50\%). Langhagen et al. [27] also found that 51\% patients with VM had a history of motion sickness. Therefore, obtaining history of motion sickness may contribute to clinical diagnosis of VM.

In this study, migraine without aura was most common in patients with VM. The most common migraine-like symptoms in VM patients was photophobia/phonophobia $(n=15,88.2 \%)$, followed by migraine-like headache $(n=8,47.1 \%)$, and visual aura $(n=7,41.2 \%)$. This result is consistent with previous studies made by Zhang et al. [28] and Cho et al. [20].

In this study, we found that compared with HCs, spontaneous functional activity in the right temporal lobe (STG and MTG), ReHo values in the right temporal lobe (STG, MTG and ITG), and FC within the right temporal lobe were both enhanced in patients with VM, suggesting enhanced regional functional activity in the right temporal lobe in patients with VM, this may be related to the mechanism of photophobia/phonophobia. A study have shown that lesions in regions within the temporal lobe such as STG (AAL; BA22), MTG, and ITG are often associated with allocentric deficits [29]. Allocentric processing is known as allocentric spatial discrimination ability, i.e., impaired stimulus-centered advanced navigation system. Medina et al. [30] performed MRI and spatial cognition tests on 171 patients with right supratentorial ischemic stroke and found that parts of the dorsal stream of visual processing, including the right supramarginal gyrus, are involved in spatial encoding in egocentric coordinates, parts of the ventral stream, including the posterior ITG, are involved in allocentric processing. Priyanka et al. [31] performed repetitive transcranial magnetic stimulation (rTMS) on the right STG $(48,-20,-8)$ in 11 right-handed healthy subjects, revealed impaired stimulus-centered spatial cognition in patients. Patients with VM in the present study are similar to healthy subjects who underwent rTMS on right STG in the above-mentioned study of Priyanka et al, indicating that ventral stream of visual processing and allocentric spatial cognition were impaired in patients with VM. We speculated that enhanced spontaneous functional activity in the right temporal lobe (STG, MTG and ITG) in patients with VM may be related to patients' discomfort and worsening of dizziness during visual stimulation. Xu et al. [32] found that MTG is associated with auditory information processing, enhanced regional functional activity of MTG in patients with VM is presumed to be related with symptoms of phonophobia

Furthermore, our findings showed that vertigo attacks in patients with VM may be associated with increased regional spontaneous activity in the right parietal lobe-frontal lobe-thalamus. (1) Compared with patients with migraine, spontaneous functional activity in the right parietal lobe (postcentral gyrus and superior parietal gyrus) $(X=24, Y=-45, Z=69)$ and right frontal lobe (SMA and dorsolateral superior frontal gyrus) $(X=24, Y=-45, Z=69)$ was enhanced in patients with VM. It is speculated that patients with VM has abnormalities in visual-spatial

Page $8 / 21$ 
information integration, and higher-level vestibular cortical integration areas are in a sensitive state. Previous studies based on working memory have shown that the dorsolateral superior frontal gyrus and superior parietal lobule (superior parietal gyrus) received visual and spatial sensory stimuli and integrate visual information flow and spatial information (vestibular information related to position) [33], the right frontal lobe is related to visualspatial tasks [34]. Generally, visual-spatial information integration requires the combined effects of top-down (cognitive control and goal-directed) modulation and bottom-up inputs (capture of external sensory stimuli) in the superior parietal lobule [35, 36]. Postle et al. [37] applied rTMS stimulation over superior parietal gyrus, and found that subjects' performance was not stable during tasks requiring visual-spatial coordination, and changes in the parietofrontal network is observed, application of rTMS on superior parietal gyrus is similar to enhanced spontaneous activity observed in patients with VM in this study. (2) Compared with patients with migraine, increase in consistency of regional brain activity in the right thalamus was observed in patients with VM. The thalamus is the relay station for all sensory input, it is also considered as an integrated area of multisensory vestibular information [38]. As mentioned above, compared with patients with migraine, VM patients showed enhanced regional functional activity in the thalamus, right parietal lobe (postcentral gyrus and superior parietal gyrus) and right frontal lobe (SMA and dorsolateral superior frontal gyrus). It is speculated that the vestibular pathway, right parietal lobe-frontal lobe-thalamus, in patients with VM is in a sensitive state, this may be related to the repeated episodes of vertigo and worsening of dizziness during visual stimulation that are different from those of patients with migraine.

In this study, we also compared the differences in the regional brain activity measures between patients with migraine and HCs. The results showed that enhanced spontaneous functional activity in the right limbic lobe (mainly including the right parahippocampal gyrus and the right fusiform gyrus) and right frontal lobe (SMA, right median cingulate and paracingulate gyri, and right inferior frontal gyrus), decreased spontaneous function activity in the pons and brainstem, as well as reduced ReHo values in the frontal cortex in patients with migraine compared with HCs. This results is consistent with the results of previous fMRI study showing that brain stem and hippocampus play a key role in the onset of migraine attack, and the limbic lobe is involved in the formation of migraine-related pain network, abnormal frontal lobe function can eventually lead to reduced pain threshold in patients with migraine [39]. Our findings showed that brain regions with altered functional activity in patients with VM are very different from those in patients with migraine relative to $\mathrm{HC}$ groups, there appears to be little overlap, and the mechanisms involved may be different.

\section{Limitations}

The limitation of the study is the small sample size, further study with larger sample size is needed to further confirm our results.

\section{Conclusions}

Our findings revealed enhanced spontaneous functional activity in the right temporal lobe (STG, MTG and ITG) in patients with VM, indicating that ventral stream of visual processing and allocentric spatial cognition may be impaired. Vertigo attacks in patients with VM may be associated with increased spontaneous activity in the right parietal lobe-frontal lobe-thalamus. Patients with VM and migraine both had altered brain function, but the mechanisms involved seem to be different. Our findings provide evidence for the regional functional properties of 
brain regions in patients with VM and for further functional connectivity studies based on ROI of multilevel vestibular pathways, as well as brain network connectome studies based on small-world brain graph theory.

\section{Abbreviations}

VM, vestibular migraine

HCs, health controls

fMRI, functional magnetic resonance imaging

PET, positron emission tomography

FC, Functional connectivity

ALFF, amplitude of low frequency fluctuation

fALFF, fractional amplitude of low frequency fluctuation

ReHo, regional homogeneity

ICHD, International Classification of Headache Disorders

RW, right warm

LW, left warm

$\mathrm{RC}$, right cold

LC, left cold

$\mathrm{CP}$, canal paresis

FWE, family-wise-error correction

SMA, supplementary motor area

STG, superior temporal gyrus

ITG, inferior temporal gyrus

MTG, middle temporal gyrus

rTMS, repetitive transcranial magnetic stimulation

\section{Declarations}

\section{Acknowledgments}

Not applicable. 


\section{Authors' contributions}

XY designed the study. ZL, LS, BS, XL performed the experiments and analyzed the data. ZL prepared the manuscript. All authors read and approved the final manuscript.

\section{Funding}

The study was supported by Aerospace Center Hospital (Grant No. YN201802).

\section{Availability of data and materials}

The datasets generated during and/or analyzed during the current study are available from the corresponding author on reasonable request.

\section{Ethics approval and consent to participate}

This study was approved by the Ethics Committee of Aerospace Center Hospital, Peking University Aerospace School of Clinical Medicine. All subjects volunteered to participate in this study and signed informed consent.

\section{Consent for publication}

Not applicable.

\section{Competing interests}

The authors declare no potential conflicts of interest with respect to the research, authorship, and/or publication of this article.

\section{Contributor Information}

Zhe-Yuan Li, Email: zheyuanli@163.com

Li-Hong Si, Email: slh931554199@163.com

Bo Shen, Email: 542068931@qq.com

Xia Ling, Email: 1515164168@qq.com

Xu Yang, Email: yangxu2011@163.com

\section{References}

1. 1. Dieterich $M$, Obermann $M$, Celebisoy $N$. Vestibular migraine: the most frequent entity of episodic vertigo. $J$ Neurol. 2016;263 Suppl 1:S82-9. doi: 10.1007/s00415-015-7905-2.

2. 2. Headache Classification Committee of the International Headache Society (IHS). The International Classification of Headache Disorders, 3rd edition (beta version). Cephalalgia. 2013;33(9):629-808. doi: $10.1177 / 0333102413485658$.

3. 3. Lempert T, Olesen J, Furman J, Waterston J, Seemungal B, Carey J, Bisdorff A, Versino M, Evers S, Newman-Toker D. Vestibular migraine: diagnostic criteria. J Vestib Res. 2012;22(4):167 - 72. doi: 
10.3233/VES-2012-0453.

4. 4. Agarwal K, Bronstein AM, Faldon ME, Mandalà M, Murray K, Silove Y. Visual dependence and BPPV. J Neurol. 2012;259(6):1117-24. doi: 10.1007/s00415-011-6311-7.

5. 5. Polensek SH, Tusa RJ. Nystagmus during attacks of vestibular migraine: an aid in diagnosis. Audiol Neurootol. 2010;15(4):241-6. doi: 10.1159/000255440.

6. 6. von Brevern $M$, Zeise $D$, Neuhauser $H$, Clarke AH, Lempert T. Acute migrainous vertigo: clinical and oculographic findings. Brain. 2005;128(Pt 2):365 - 74. doi: 10.1093/brain/awh351.

7. 7. Balaban CD. Migraine, vertigo and migrainous vertigo: Links between vestibular and pain mechanisms. J Vestib Res. 2011;21(6):315 - 21. doi: 10.3233/VES-2011-0428.

8. 8. Furman JM, Marcus DA, Balaban CD. Vestibular migraine: clinical aspects and pathophysiology. Lancet Neurol. 2013;12(7):706 - 15. doi: 10.1016/S1474-4422(13)70107-8.

9. 9. Wang J, Lewis RF. Abnormal Tilt Perception During Centrifugation in Patients with Vestibular Migraine. J Assoc Res Otolaryngol. 2016;17(3):253-8. doi: 10.1007/s10162-016-0559-7.

10. 10. Shin JH, Kim YK, Kim HJ, Kim JS. Altered brain metabolism in vestibular migraine: comparison of interictal and ictal findings. Cephalalgia. 2014;34(1):58-67. doi: 10.1177/0333102413498940.

11. 11. Bottini G, Karnath HO, Vallar G, Sterzi R, Frith CD, Frackowiak RS, Paulesu E. Cerebral representations for egocentric space: Functional-anatomical evidence from caloric vestibular stimulation and neck vibration. Brain. 2001;124(Pt 6):1182-96. doi: 10.1093/brain/124.6.1182.

12. 12. Russo A, Marcelli V, Esposito F, Corvino V, Marcuccio L, Giannone A, Conforti R, Marciano E, Tedeschi G, Tessitore A. Abnormal thalamic function in patients with vestibular migraine. Neurology. 2014;82(23):2120-6. doi: 10.1212/WNL.0000000000000496.

13. 13. Bense S, Stephan T, Yousry TA, Brandt T, Dieterich M. Multisensory cortical signal increases and decreases during vestibular galvanic stimulation (fMRI). J Neurophysiol. 2001;85(2):886 - 99. doi: 10.1152/jn.2001.85.2.886.

14. 14. Bucher SF, Dieterich M, Wiesmann M, Weiss A, Zink R, Yousry TA, Brandt T. Cerebral functional magnetic resonance imaging of vestibular, auditory, and nociceptive areas during galvanic stimulation. Ann Neurol. 1998;44(1):120-5. doi: 10.1002/ana.410440118.

15. 15. Lee $M H$, Smyser CD, Shimony JS. Resting-state fMRI: a review of methods and clinical applications. AJNR Am J Neuroradiol. 2013;34(10):1866-72. doi: 10.3174/ajnr.A3263.

16. 16. Headache Classification Committee of the International Headache Society (IHS). The International Classification of Headache Disorders, 3rd edition (beta version). Cephalalgia. 2013;33(9):629-808. doi: $10.1177 / 0333102413485658$.

17. 17. Caloric test protocol. Br J Audiol. 1999;33(3):179 - 84.

18. 18. Stolte B, Holle D, Naegel S, Diener HC, Obermann M. Vestibular migraine. Cephalalgia. 2015;35(3):262 70. doi: $10.1177 / 0333102414535113$.

19. 19. Neuhauser HK, Radtke A, von Brevern M, Feldmann M, Lezius F, Ziese T, Lempert T. Migrainous vertigo: prevalence and impact on quality of life. Neurology. 2006;67(6):1028-33. doi:

10.1212/01.wnl.0000237539.09942.06.

20. 20. Cho SJ, Kim BK, Kim BS, Kim JM, Kim SK, Moon HS, Song TJ, Cha MJ, Park KY, Sohn JH. Vestibular migraine in multicenter neurology clinics according to the appendix criteria in the third beta edition of the 
International Classification of Headache Disorders. Cephalalgia. 2016;36(5):454 - 62. doi: $10.1177 / 0333102415597890$.

21. 21. Boldingh MI, Ljøstad U, Mygland A, Monstad P. Vestibular sensitivity in vestibular migraine: VEMPs and motion sickness susceptibility. Cephalalgia. 2011;31(11):1211-9. doi: 10.1177/0333102411409074.

22. 22. Heuberger M, Sağlam M, Todd NS, Jahn K, Schneider E, Lehnen N. Covert anti-compensatory quick eye movements during head impulses. PLoS One. 2014;9(4):e93086. doi: 10.1371/journal.pone.0093086.

23. 23. Teggi R, Colombo B, Bernasconi L, Bellini C, Comi G, Bussi M. Migrainous vertigo: results of caloric testing and stabilometric findings. Headache. 2009;49(3):435 - 44. doi: 10.1111/j.1526-4610.2009.01338.x.

24. 24. Jeong SH, Oh SY, Kim HJ, Koo JW, Kim JS. Vestibular dysfunction in migraine: effects of associated vertigo and motion sickness. J Neurol. 2010;257(6):905 - 12. doi: 10.1007/s00415-009-5435-5.

25. 25. Kayan A, Hood JD. Neuro-otological manifestations of migraine. Brain. 1984;107 (Pt 4):1123-42. doi: 10.1093/brain/107.4.1123.

26. 26. Akdal G, Baykan B, Ertaş M, Zarifoğlu M, Karli N, Saip S, Siva A. Population-based study of vestibular symptoms in migraineurs. Acta Otolaryngol. 2015;135(5):435-9. doi: 10.3109/00016489.2014.969382.

27. 27. Langhagen T, Lehrer N, Borggraefe I, Heinen F, Jahn K. Vestibular migraine in children and adolescents: clinical findings and laboratory tests. Front Neurol. 2015;5:292. doi: 10.3389/fneur.2014.00292.

28. 28. Zhang Y, Kong Q, Chen J, Li L, Wang D, Zhou J. International Classification of Headache Disorders 3rd edition beta-based field testing of vestibular migraine in China: Demographic, clinical characteristics, audiometric findings and diagnosis statues. Cephalalgia. 2016;36(3):240-8. doi:

$10.1177 / 0333102415587704$.

29. 29. Grimsen C, Hildebrandt H, Fahle M. Dissociation of egocentric and allocentric coding of space in visual search after right middle cerebral artery stroke. Neuropsychologia. 2008;46(3):902 - 14. doi: 10.1016/j.neuropsychologia.2007.11.028.

30. 30. Medina J, Kannan V, Pawlak MA, Kleinman JT, Newhart M, Davis C, Heidler-Gary JE, Herskovits EH, Hillis AE. Neural substrates of visuospatial processing in distinct reference frames: evidence from unilateral spatial neglect. J Cogn Neurosci. 2009;21(11):2073-84. doi: 10.1162/jocn.2008.21160.

31. 31. Shah-Basak PP, Chen P, Caulfield K, Medina J, Hamilton RH. The role of the right superior temporal gyrus in stimulus-centered spatial processing. Neuropsychologia. 2018;113:6-13. doi:

10.1016/j.neuropsychologia.2018.03.027.

32. 32. Xu J, Lyu H, Li T, Xu Z, Fu X, Jia F, Wang J, Hu Q. Delineating functional segregations of the human middle temporal gyrus with resting-state functional connectivity and coactivation patterns. Hum Brain Mapp. 2019;40(18):5159-5171. doi: 10.1002/hbm.24763.

33. 33. Koenigs M, Barbey AK, Postle BR, Grafman J. Superior parietal cortex is critical for the manipulation of information in working memory. J Neurosci. 2009;29(47):14980-6. doi: 10.1523/JNEUROSCI.3706-09.2009.

34. 34. Koenigs M, Acheson DJ, Barbey AK, Solomon J, Postle BR, Grafman J. Areas of left perisylvian cortex mediate auditory-verbal short-term memory. Neuropsychologia. 2011;49(13):3612-9. doi:

10.1016/j.neuropsychologia.2011.09.013.

35. 35. Pinto Y, van der Leij AR, Sligte IG, Lamme VA, Scholte HS. Bottom-up and top-down attention are independent. J Vis. 2013;13(3):16. doi: 10.1167/13.3.16.

36. 36. Wu Z, Mazzola CA, Catania L, Owoeye O, Yaramothu C, Alvarez T, Gao Y, Li X. Altered cortical activation and connectivity patterns for visual attention processing in young adults post-traumatic brain injury: A 
functional near infrared spectroscopy study. CNS Neurosci Ther. 2018;24(6):539-548. doi:

$10.1111 /$ cns. 12811.

37. 37. Postle BR, Ferrarelli F, Hamidi M, Feredoes E, Massimini M, Peterson M, Alexander A, Tononi G. Repetitive transcranial magnetic stimulation dissociates working memory manipulation from retention functions in the prefrontal, but not posterior parietal, cortex. J Cogn Neurosci. 2006;18(10):1712-22. doi:

10.1162/jocn.2006.18.10.1712.

38. 38. Brandt T, Dieterich M. Thalamocortical network: a core structure for integrative multimodal vestibular functions. Curr Opin Neurol. 2019;32(1):154-164. doi: 10.1097/WC0.0000000000000638.

39. 39. Russo A, Silvestro M, Tedeschi G, Tessitore A. Physiopathology of Migraine: What Have We Learned from Functional Imaging? Curr Neurol Neurosci Rep. 2017;17(12):95. doi: 10.1007/s11910-017-0803-5.

\section{Tables}

Table 1 Clinical characteristics of patients with patients with vestibular migraine

\begin{tabular}{|c|c|c|c|c|c|c|c|}
\hline \multirow[t]{2}{*}{ ID/sex/age } & \multirow{2}{*}{$\begin{array}{l}\text { Recurrent } \\
\text { spontaneous } \\
\text { vertigo }\end{array}$} & \multicolumn{3}{|c|}{ Accompanying migraine features } & \multicolumn{2}{|l|}{ Caloric test } & \multirow{2}{*}{$\begin{array}{l}\text { Motion } \\
\text { sickness }\end{array}$} \\
\hline & & $\begin{array}{l}\text { Photophobia } \\
\text { /Phonophobia }\end{array}$ & $\begin{array}{l}\text { Migraine- } \\
\text { like } \\
\text { headache }\end{array}$ & $\begin{array}{l}\text { Visual } \\
\text { aura }\end{array}$ & $\begin{array}{l}\text { Hyperactive } \\
\text { response }\end{array}$ & $\begin{array}{l}\text { Caloric } \\
\text { test } \\
\text { intolerance }\end{array}$ & \\
\hline 1/F/52 & & $\sqrt{ }$ & & & & & \\
\hline $2 / \mathrm{M} / 24$ & $\sqrt{ }$ & $\sqrt{ }$ & $\sqrt{ }$ & $\sqrt{ }$ & & $\sqrt{ }$ & $\sqrt{ }$ \\
\hline $3 / F / 30$ & $\sqrt{ }$ & $\sqrt{ }$ & & & & & $\sqrt{ }$ \\
\hline $4 / \mathrm{M} / 47$ & $\sqrt{ }$ & $\sqrt{ }$ & & & & $\sqrt{ }$ & $\sqrt{ }$ \\
\hline $5 / \mathrm{M} / 40$ & $\sqrt{ }$ & $\sqrt{ }$ & & & & $\sqrt{ }$ & $\sqrt{ }$ \\
\hline $6 / M / 25$ & & $\sqrt{ }$ & $\sqrt{ }$ & & $\sqrt{ }$ & $\sqrt{ }$ & $\sqrt{ }$ \\
\hline $7 / F / 38$ & $\sqrt{ }$ & $\sqrt{ }$ & $\sqrt{ }$ & $\sqrt{ }$ & & $\sqrt{ }$ & $\sqrt{ }$ \\
\hline 8/M/39 & & $\sqrt{ }$ & & & $\sqrt{ }$ & $\sqrt{ }$ & \\
\hline $9 / F / 35$ & $\sqrt{ }$ & $\sqrt{ }$ & & & & $\sqrt{ }$ & \\
\hline 10/F/34 & & $\sqrt{ }$ & $\sqrt{ }$ & $\sqrt{ }$ & $\sqrt{ }$ & $\sqrt{ }$ & $\sqrt{ }$ \\
\hline 11/F/58 & $\sqrt{ }$ & & $\sqrt{ }$ & & & & $\sqrt{ }$ \\
\hline 12/M/35 & $\sqrt{ }$ & $\sqrt{ }$ & & & & $\sqrt{ }$ & \\
\hline $13 / F / 47$ & $\sqrt{ }$ & $\sqrt{ }$ & $\sqrt{ }$ & $\sqrt{ }$ & $\sqrt{ }$ & $\sqrt{ }$ & $\sqrt{ }$ \\
\hline $14 / F / 54$ & $\sqrt{ }$ & $\sqrt{ }$ & $\sqrt{ }$ & $\sqrt{ }$ & $\sqrt{ }$ & $\sqrt{ }$ & $\sqrt{ }$ \\
\hline $15 / F / 44$ & & $\sqrt{ }$ & & & & & \\
\hline $16 / F / 38$ & $\sqrt{ }$ & $\sqrt{ }$ & & $\sqrt{ }$ & & & $\sqrt{ }$ \\
\hline 17/M/31 & $\sqrt{ }$ & & $\sqrt{ }$ & $\sqrt{ }$ & & $\sqrt{ }$ & \\
\hline
\end{tabular}


The criteria for hyperactive responses were as follows: total peak cool response $\varangle \mathrm{LC}+\mathrm{RC} \otimes$ of $>99^{\circ} / \mathrm{s}$, total peak warm response $\varangle \mathrm{LW}+\mathrm{RW} \rrbracket$ of $>146^{\circ} / \mathrm{s}$, total peak response $\varangle \mathrm{LC}+\mathrm{RC}+\mathrm{LW}+\mathrm{RW} \bigotimes$ of $>221 \%$ s. Caloric test intolerance refers to the main symptoms, including obvious nausea, vomiting, numbness in hands and feet, and body stiffness. Caloric test intolerance was considered severe if its duration was $>1 \mathrm{~h}$.

Table 2 Altered regional brain functional activity in patients with VM measured by fMRI 


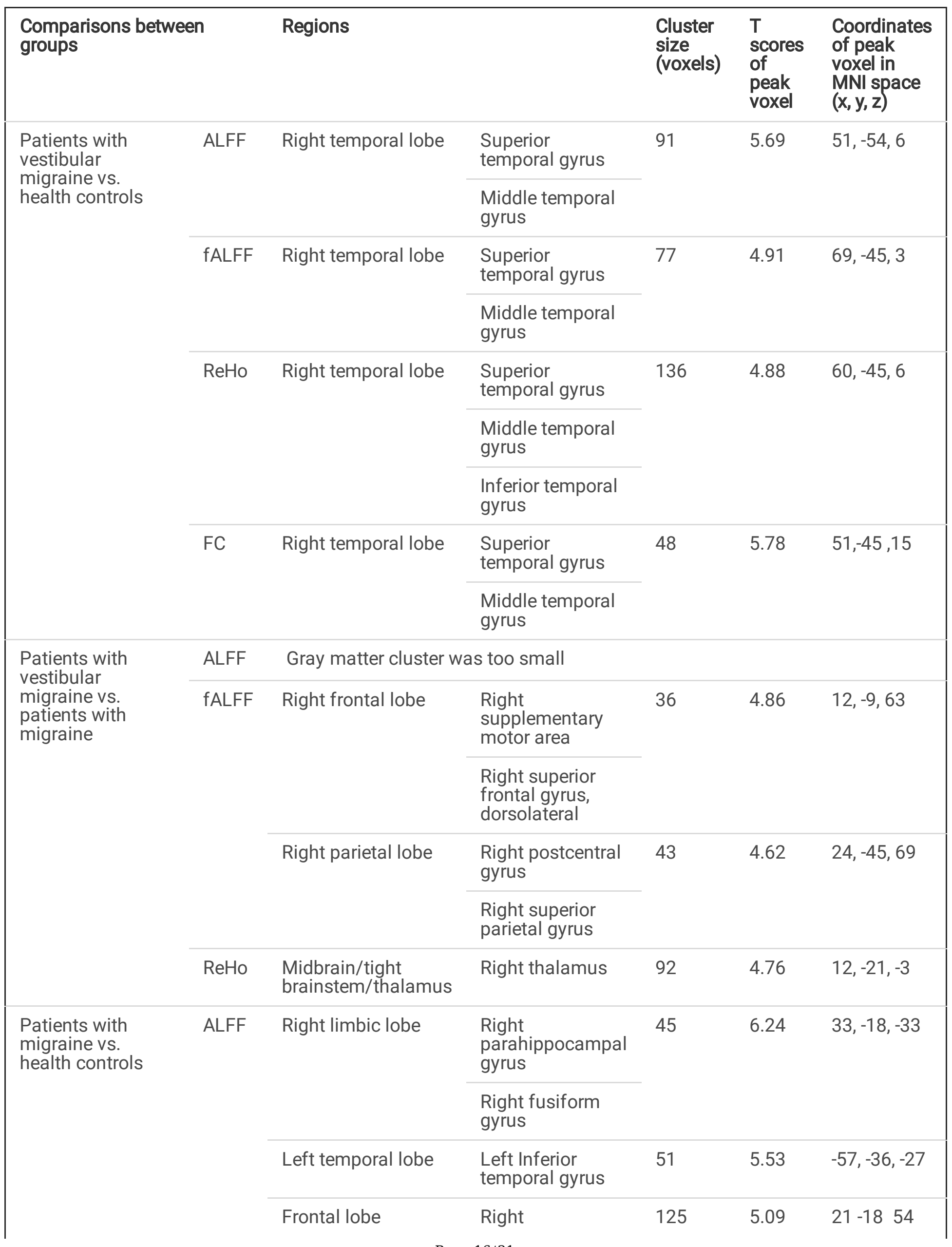

Page 16/21 


\begin{tabular}{|c|c|c|c|c|c|}
\hline & \multirow[b]{4}{*}{ Pons\& brainstem } & \multirow{2}{*}{$\begin{array}{l}\text { supplementary } \\
\text { motor area } \\
\text { Median cingulate } \\
\text { and } \\
\text { paracingulate } \\
\text { gyri }\end{array}$} & & \multirow[b]{4}{*}{-5.56} & \multirow[b]{4}{*}{$6,-33,-33$} \\
\hline & & & & & \\
\hline & & \multicolumn{2}{|l|}{$\begin{array}{l}\text { Right superior } \\
\text { frontal gyrus, } \\
\text { dorsolateral }\end{array}$} & & \\
\hline & & Pons\& brainstem & 58 & & \\
\hline fALFF & \multicolumn{5}{|c|}{ Gray matter cluster was too small } \\
\hline \multirow[t]{7}{*}{ ReHo } & \multirow[t]{7}{*}{ Frontal lobe } & $\begin{array}{l}\text { Right } \\
\text { supplementary } \\
\text { motor area }\end{array}$ & \multirow[t]{7}{*}{737} & \multirow[t]{7}{*}{-9.62} & \multirow[t]{7}{*}{$-18,3,60$} \\
\hline & & $\begin{array}{l}\text { Left } \\
\text { supplementary } \\
\text { motor area }\end{array}$ & & & \\
\hline & & $\begin{array}{l}\text { Left median } \\
\text { cingulate and } \\
\text { paracingulate } \\
\text { gyri }\end{array}$ & & & \\
\hline & & $\begin{array}{l}\text { Right paracentral } \\
\text { lobule }\end{array}$ & & & \\
\hline & & $\begin{array}{l}\text { Right superior } \\
\text { frontal gyrus, } \\
\text { dorsolateral }\end{array}$ & & & \\
\hline & & $\begin{array}{l}\text { Right middle } \\
\text { frontal gyrus }\end{array}$ & & & \\
\hline & & $\begin{array}{l}\text { Left middle } \\
\text { frontal gyrus }\end{array}$ & & & \\
\hline
\end{tabular}

ALFF, amplitude of low frequency fluctuation; fALFF, fractional amplitude of low frequency fluctuation; ReHo, regional homogeneity

\section{Figures}



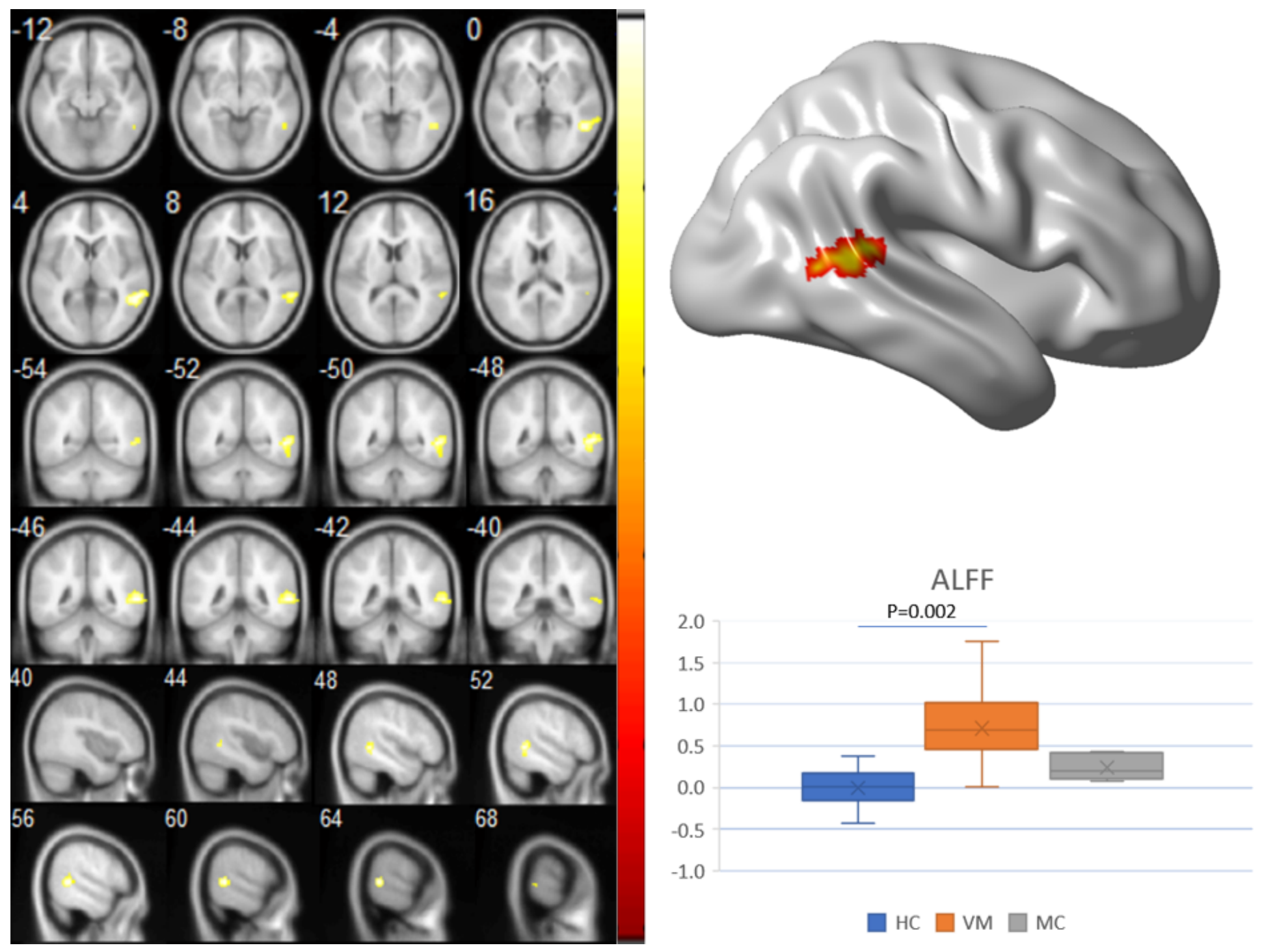

Figure 1

Differences in ALFF values between three groups. Vestibular migraine (VM) group exhibited significantly increased ALFF values in the right temporal lobe compared with health control (HC) group $(X=51, Y=-54, Z=6)$, indicating enhanced spontaneous functional activity in the right temporal lobe in patients with VM. No significant difference was found between migraine and HC groups, as well as VM and migraine groups. 


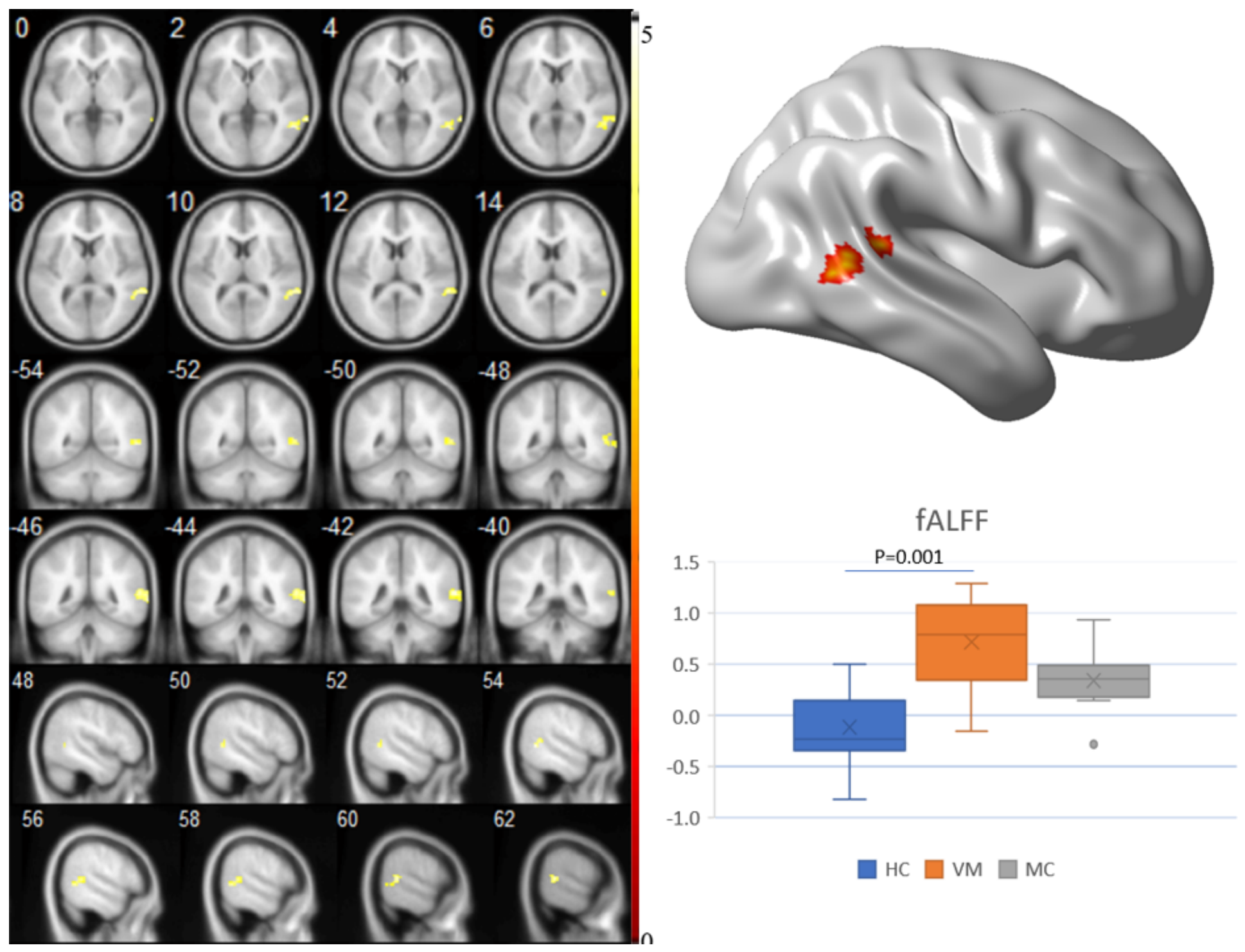

Figure 2

Differences in fALFF values between three groups. Vestibular migraine (VM) group had significantly increased fALFF values in the right temporal lobe compared with health control $(\mathrm{HC})$ group $(X=69, Y=-45, Z=3)$, indicating that spontaneous functional activity in this area was enhanced in patients with VM, which corroborated the ALFF results. No significant difference was found between migraine and $\mathrm{HC}$ groups, as well as VM and migraine groups. 

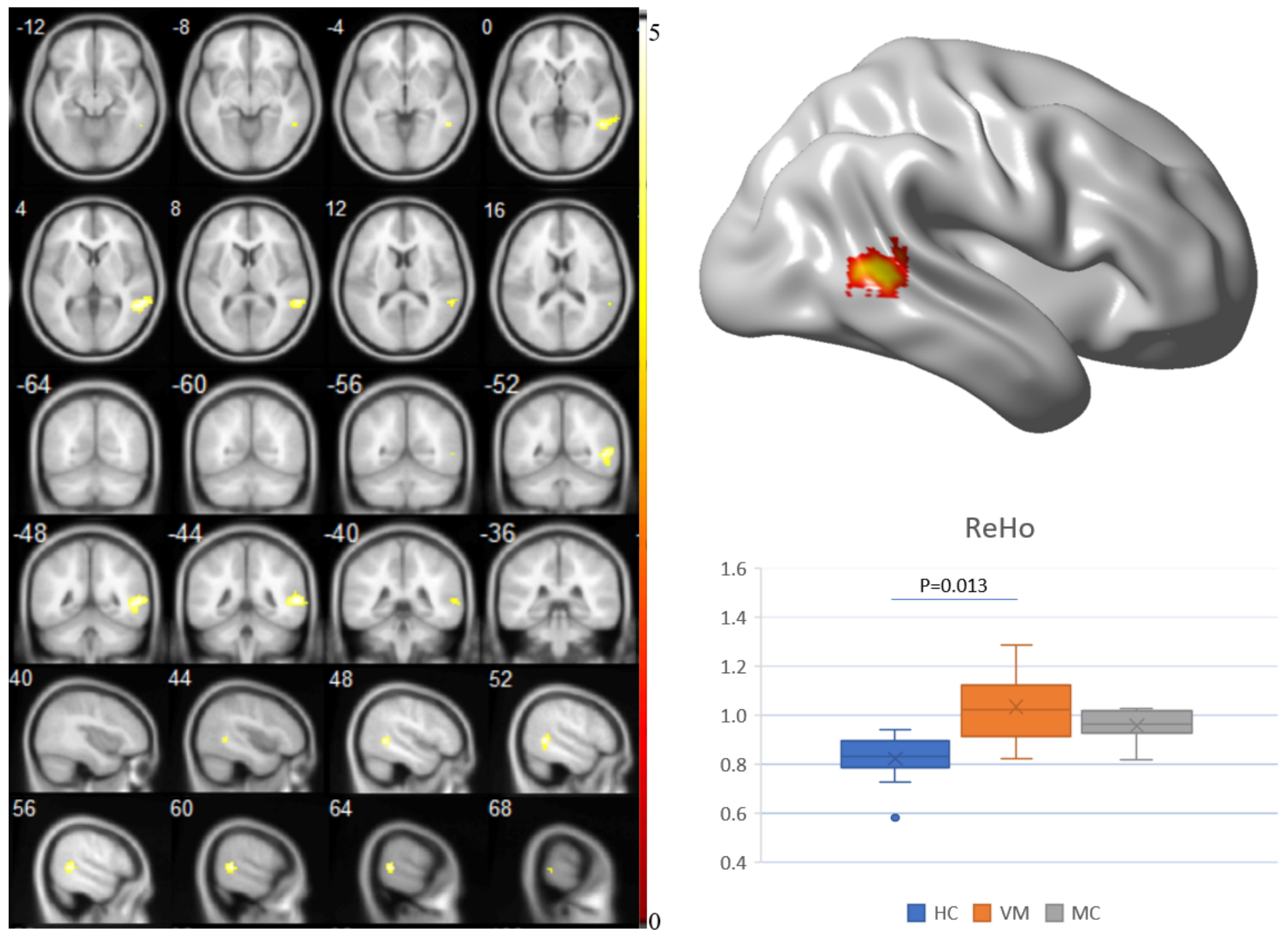

Figure 3

Differences in ReHo values between the three groups. Vestibular migraine (VM) group showed significantly increased ReHo values in the right temporal lobe, including superior temporal gyrus (STG) and middle temporal gyrus (MTG) and inferior temporal gyrus (ITG), compared with health control (HC) group ( $X=60, Y=-45, Z=6$, $\mathrm{P}=0.013$, FWE corrected). No significant difference was found between migraine and HC groups, as well as VM and migraine groups. 

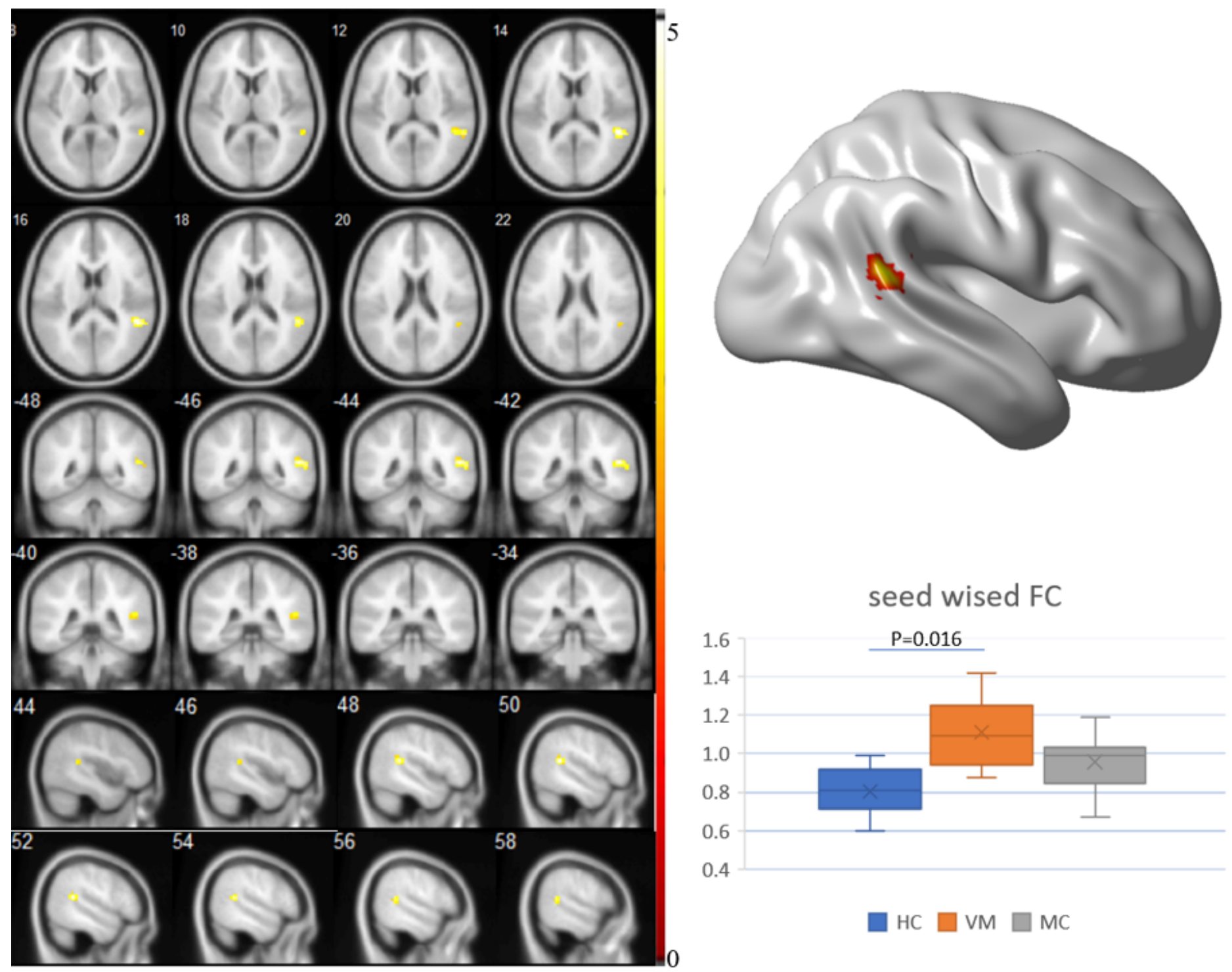

Figure 4

Differences in functional connectivity (FC) between the three groups. Vestibular migraine (VM) group showed significantly increased $F C$ in the right temporal lobe $(X=51, Y=-45, Z=15)$ compared with health control (HC) group, indicating that functional connectivity between this region and the seed regions was enhanced $(X=69, Y=-45, Z=3)$, which corroborated the ReHo results. No significant difference was found between migraine and HC groups, as well as VM and migraine groups. 